\title{
Variaciones Anatómicas Radiculares y Sistemas de Canales
}

\author{
Tooth Root and Pulp Canal Anatomical Variations
}

Gonzalo H. Oporto V.; Ramón E. Fuentes F. \& Camila C. Soto P.

\begin{abstract}
OPORTO, V. G. H.; FUENTES, F. R. E. \& SOTO, P. C. C. Variaciones anatómicas radiculares y sistemas de canales. Int. J. Morphol., 28(3):945-950, 2010.

RESUMEN: El desarrollo dentario resulta de una complicada interacción entre el epitelio oral y el tejido mesenquimático subyacente durante la Odontogénesis. La interacción anormal de estos tejidos podría resultar en el desarrollo y/o erupción de un diente anómalo, ectópico o con variación anatómica. El presente reporte muestra tres casos de variaciones anatómicas radiculares y al sistema de canales: un canino mandibular, un premolar mandibular y un primer premolar maxilar. El diagnóstico en los tres casos se efectuó por medio del análisis de radiografías. El canino y premolar mandibular presentaron dos raíces y dos canales, el premolar maxilar presentó tres raíces y tres conductos. Dada la presencia de una caries penetrante en este último, fue derivado a un especialista en endodoncia para su tratamiento y posterior rehabilitación. Idealizar el sistema de canales como una entidad simple es un error, por lo que es importante considerar estas variaciones anatómicas especialmente ante presencia de dientes con patologías que requieran tratamientos de endodoncia o cualquier procedimiento al interior del sistema de canales, ya que la oportuna y adecuada indicación de exámenes complementarios permite pesquisar y diagnosticar variaciones anatómicas de este tipo, para minimizar la ocurrencia de accidentes durante el tratamiento.
\end{abstract}

PALABRAS CLAVE: variación anatómica, canal radicular, raíz dentaria.

\section{INTRODUCTION}

El desarrollo dentario resulta de una complicada interacción entre el epitelio oral y el tejido mesenquimático subyacente durante la Odontogénesis (Fuentes \& Oporto, 2009; Goh, 2001). Es así como una vez que ha finalizado la formación de la corona, comienza el desarrollo radicular (Abramovich, 1999). La embriología de los tejidos de la raíz del diente es promovido por el complejo epiteliomesénquima, mediante interacciones célula-célula y célulamatriz extracelular. Gran número de aspectos de la histología dental tales como formación de los dientes, determinación de la forma coronaria, iniciación de la formación de dentina, cemento y los otros tejidos, además de la formación radicular, dependen de estas relaciones (Ten Cate, 1986).

Las variaciones morofológicas ocurren por la interacción anormal de tejidos durante las señaladas fases del desarrollo dentario. De acuerdo al periodo en que ocurra esta alteración, el diente podría evidenciar un desarrollo y/o erupción anómala, ectópico o con alguna variación anatómica.

Las teorías que explican la existencia de un número de raíces superior a lo normal, incluyen una hiperactividad de la vaina de Hertwig (Holtzman, 1997) y una degeneración patológica en un área de la vaina epitelial radicular de Hertwig, lo cual produce una invaginación por parte de la papila dental, formándose así una raíz accesoria (Roig \& Morelló, 2006).

Se ha reportado una amplia gama de variaciones anatómicas en el sistema de conductos, donde se ha estudiado que el número de estos, su anatomía e interconexiones, varían entre sí según grupo dentario.

En el caso del canino mandibular, este es usualmente considerado como una pieza unirradicular y que contiene sólo un conducto acompañando a ésta, situación que se ha reportado en aproximadamente el $93,48 \%$. Aunque es posible encontrar caninos con más de un conducto radicular (Sharma et al., 1998), sólo un $15 \%$ de los caninos mandibulares presenta dos conductos radiculares y una raíz (con uno o dos forámenes apicales). Y tan solo el $1 \%$ de los reportes, corresponden a piezas con dos raíces y dos canales (Victorino et al., 2009). 
Por otro lado, Soares y Leonardo (2003) resaltan que los premolares superiores presentan una morfología de los conductos radiculares altamente variable. Entre las variables morfológicas de este grupo de dientes, se encuentra la posibilidad de presencia de tres conductos radiculares y tres raíces. Un $61 \%$ de los casos presenta 2 raíces: una vestibular y otra palatina. El 35,5\% de los casos puede presentar una raíz, y en porcentaje mucho menor $(3,5 \%)$ tres raíces; dos vestibulares y una palatina, cuyos conductos se vuelven estrechos. En un $84.2 \%$ presenta dos conductos radiculares, incluso cuando presenta una sola raíz, caracterizándose por ser conductos estrechos pero rectos (Soares \& Goldberg, 2002).

Con respecto a los primeros premolares mandibulares, en su mayoría presentan una raíz con conducto único, bien centrado. Ingle y colaboradores (1987) sostienen que el primer premolar inferior presenta un conducto y un forámen apical en un $73.5 \%$ de los casos, dos conductos y un foramen apical en un $6.5 \%$, dos conductos y dos forámenes en un $19.5 \%$ y tres conductos en un $0.5 \%$.

De esta manera, el conocimiento de los reparos anatómicos normales de los conductos radiculares y de sus posibles variaciones, es imprescindible para tener una práctica profesional exitosa y minimizar la posibilidad de ocurrencia de accidentes durante el desarrollo de tratamientos odontológicos.

Se describen tres casos clínicos (un canino y dos premolares) con variaciones a la anatomía radicular y al sistema de conductos.

\section{MATERIAL Y MÉTODO}

Se reportan tres casos de dientes (caninos y pre molares) con variaciones en la morfología radicular y de la anatomía del sistema de conductos en pacientes que consultaron espontáneamente solicitando atención odontológica Integral.

El diagnóstico en los tres casos se efectuó durante una evaluación por medio del análisis de radiografías. Uno de los dientes alterados correspondió a un canino mandibular birradicular, cuya pesquisa se efectuó a través de una radiografía retroalveolar en la Clínica Odontológica Docente Asistencial de la Facultad de Medicina de la Universidad de La Frontera. Los otros dientes con variaciones correspondieron a premolares, uno maxilar y otro mandibular. Estas piezas fueron estudiadas mediante una radiografía panorámica en la Clínica Odontológica de la Cruz Roja Chilena
Filial Temuco. Luego de realizar el hallazgo, la paciente fue derivada nuevamente para la toma de nuevas radiografías, esta vez retroalveolares de ambos dientes alterados.

Posteriormente, las radiografías fueron digitalizadas sobre un negatoscopio mediante la toma de fotografías con una cámara fotográfica digital réflex Nikon D - 90 con Lente Macro de $105 \mathrm{~mm}$. sin utilizar flash. Las imágenes no fueron retocadas por medio de software, sólo encuadradas.

\section{RESULTADOS}

Reporte de canino mandibular birradicular. Uno de los dientes estudiados correspondió a un canino mandibular izquierdo, y fue un hallazgo realizado en una paciente de género femenino de 37 años de edad que consultó espontáneamente por tratamiento odontológico integral en las dependencias de la Clínica Odontológica Docente Asistencial de la Facultad de Medicina de la Universidad de La Frontera.

Durante la anamnesis, la paciente no relató poseer patología de base de larga data, y no manifestó poseer familiares cercanos con antecedentes mórbidos de relevancia. La paciente se encontraba en buen estado de salud. Hizo énfasis en que poseía varios tratamientos de endodoncia realizados, de larga data.

$\mathrm{Al}$ examen extra oral no fueron evidenciadas alteraciones en relación al diente en estudio.

Durante la exploración intra oral, se evidenció gingivitis asociada a placa bacteriana marginopapilar generalizada, múltiples lesiones de caries, presencia de restauraciones desajustadas y con recidiva de caries.

En consideración a la importante cantidad de lesiones de caries y restauraciones que requerían re tratamiento, sumado a su historia de terapia endodóntica, fueron indicadas radiografía retroalveolar total.

El diagnóstico del canino birradicular se efectuó durante el análisis radiográfico. En la toma radiográfica en posición ortoradial (Fig.1) fue posible observar un doble contorno en la zona periapical, lo que hizo sospechar la posible presencia de un canino mandibular birradicular. Se solicitó una radiografía retroalveolar por deslizamiento, la que confirmó la presencia de un canino mandibular izquierdo con dos raíces y además dos conductos radiculares (Fig. 2). Cabe señalar que durante el examen intra oral, la pieza dentaria no llamó la atención del clínico en virtud de no presentar alteraciones de la morfología en la corona clínica, 


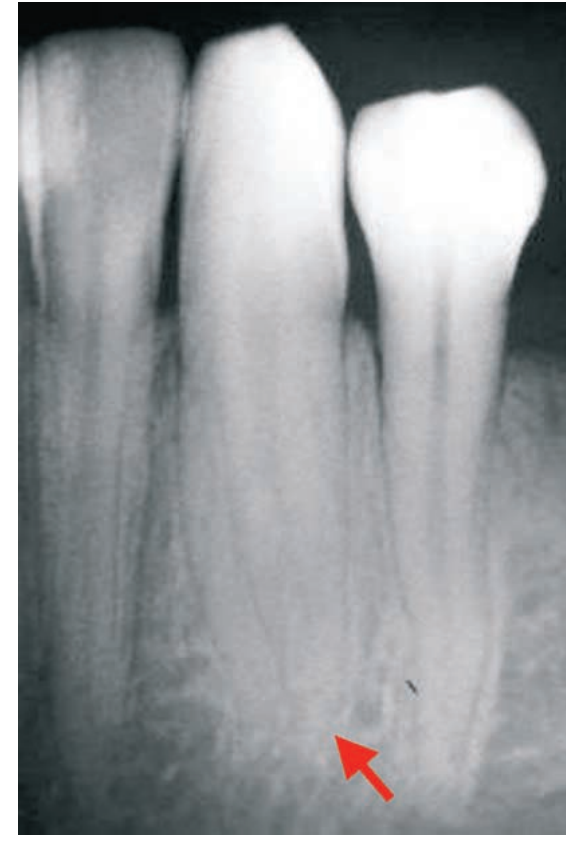

Fig. 1. Orthoradial X-ray of two-root canine (tooth 3.3). Notice the double apical contour (red arrow)

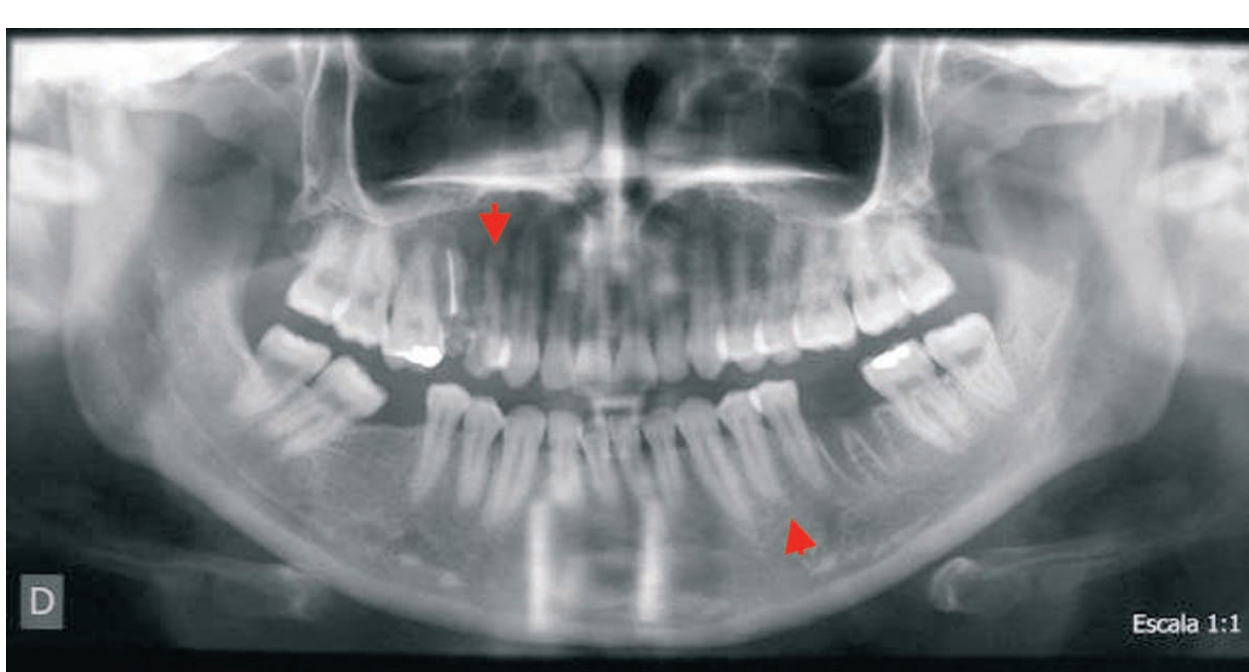

Fig. 3. Panoramic X-ray where anatomical alterations can be observed in teeth 1.4 and 3.4

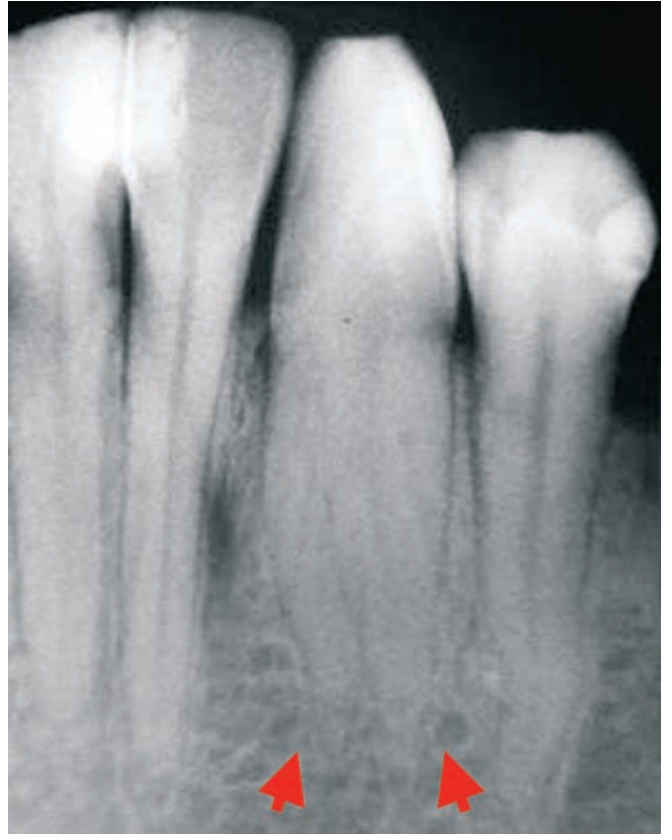

Fig. 2. Sliding X-ray of two-root canine (tooth 3.3). Notice the presence of two roots and two Canals (red arrows) no se ubicó en mal posición y no se encontró afectada por ningún tipo de patología. Radiográficamente, no evidenció imagen compatible con alteración de la normalidad distinta de la ya expuesta.

\section{Reporte de primer premolar maxilar trirradicular y pri-} mer premolar mandibular birradicular. Los dos restantes casos de dientes con alteraciones a la anatomía fueron encontrados en una paciente de género femenino de 30 años de edad que consultó espontáneamente por tratamiento de rehabilitación oral sobre prótesis fija en las dependencias de la Clínica Odontológica de la Cruz Roja Chilena Filial Temuco.

En la anamnesis, no relató poseer patología de base alguna, como tampoco antecedentes mórbidos familiares. La paciente se encontraba sana. Al examen extra oral todos los parámetros se encontraron dentro de rangos de normalidad.

Durante la exploración intra oral, se evidenció gingivitis asociada a placa bacteriana marginopapilar generalizada, ausencia de pieza 4.6, alvéolo de pieza 3.6 en organi- 


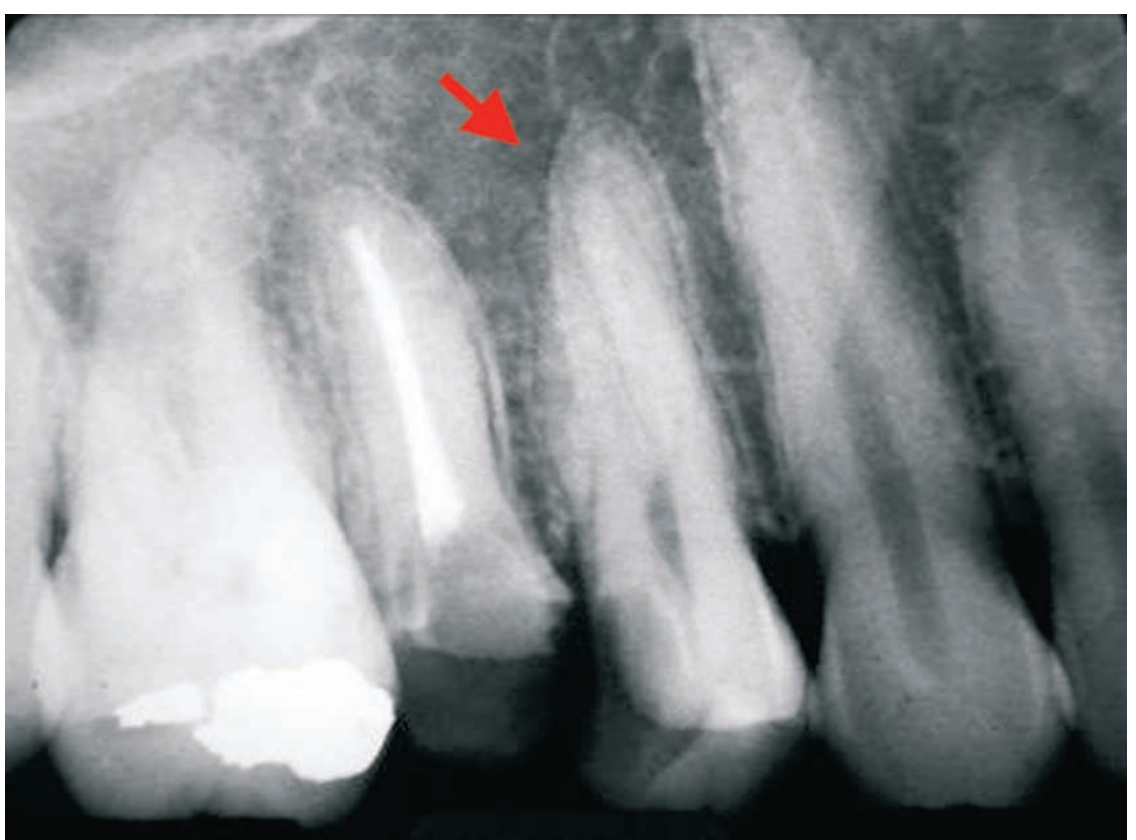

Fig. 4. Retroalveolar X-ray tooth 1.4. Be noticed that the piece has three roots and three root canals.

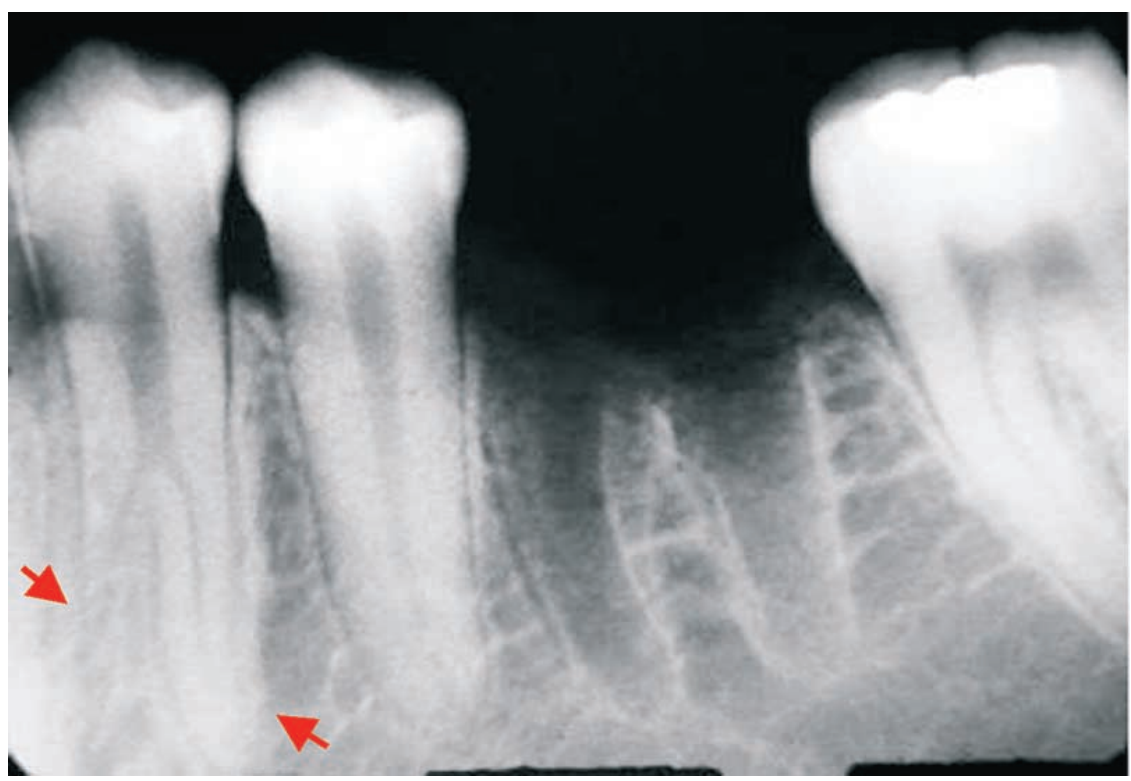

Fig. 5. Retroalveolar X-ray tooth 3.4. Be noticed it has two roots and two root canals.

zación, múltiples lesiones de caries amelo dentinarias, piezas 1.4 y 1.5 caries penetrante, presencia de restauraciones desajustadas y con recidiva de caries.

En virtud del motivo de consulta, se solicitó una radiografía panorámica para la planificación inicial de prótesis Implantoasistida de las piezas 3.6 y 4.6, además se solicitó radiografía retroalveolar de las piezas 1.4, 1.5.

Durante el análisis de la radiografía panorámica, se observó claramente la conformación trirradicular de la pieza 1.4 (Fig. 3), lo que se confirmó a la visuali- zación de la radiografía retroalveolar del mismo diente, donde además se pesquisó la presencia de tres conductos radiculares (Fig. 4). Dado que radiográficamente la lesión de caries se encontraba próxima a cámara pulpar, fue derivada para realizar tratamiento de endodoncia a un especialista en el área, para luego rehabilitar con prótesis fija singular.

En el análisis de la radiografía panorámica, se evidenció un doble contorno en la zona periapical de la pieza 3.4, situación que hizo sospechar la presencia de un premolar inferior birradicular (Fig. 3). Para complementar el estudio, se solicitó una radiografía retroalveolar, en la cual fue posible establecer claramente la presencia de dos raíces y dos conductos radiculares (Fig. 5). No evidenció imagen compatible con Patología. En consideración a que durante el examen intraoral la pieza dentaria no presentó alteraciones de la morfología en la corona clínica y no se encontraba afectada por caries u otro tipo de enfermedad, esta no fue intervenida.

\section{DISCUSIÓN}

Como se conoce, una vez que ha finalizado la formación de la corona del diente, comienza el desarrollo de la región radicular (Abramovich). El desarrollo embriológico de los tejidos de la raíz del diente es promovido por el complejo epiteliomesénquima, mediante interacciones célula-célula y célula-matriz extracelular. Muchas características de la histología dental, incluyendo la formación de los dientes, la determinación de la forma de la corona dentaria, la iniciación de la formación de dentina, cemento y los otros tejidos constituyentes de la estructura dental, como además de la formación radicular dependen de estas relaciones (Ten Cate). 
En este caso, las teorías que explican la existencia de un número de raíces superior a lo normal, incluyen una hiperactividad de la vaina de Hertwig (Roig \& Morelló), y una degeneración patológica en un área de la vaina epitelial radicular de Hertwig, lo cual produce una invaginación por parte de la papila dental, formándose así una raíz accesoria (Kitamura).

Como ya se mencionó, el diagnóstico de raíces y conductos radiculares se realiza mediante el análisis radiográfico. Ingle et al. (1996), establecieron la dificultad que existe en detectar la presencia de estas raíces supernumerarias, por lo que señalaron que al observar un contorno radicular poco claro o fuera de lo común, debe sospecharse de la presencia de una raíz accesoria (Kitamura). Aún cuando hay que tomar en cuenta que las bifurcaciones en la zona cervical o tercio medio de la raíz se puede observar sólo cuando el ángulo de incidencia de los rayos no causa superposición de imágenes. En los casos expuestos, y en conformidad con la afirmación de Ingle \& Backland (1996) la identificación de las raíces supernumerarias fue evidente observación de los contornos radiculares.

Como se enunció previamente, las variaciones anatómicas expuestas son poco frecuentes. Sólo un $1 \%$ de los caninos mandibulares corresponden a piezas con dos raíces y dos canales (Soares \& Leonardo), un 3,5\% de los premolares maxilares presenta tres raíces (Soares \& Goldberg) y los primeros premolares mandibulares poseen dos conductos y dos forámenes apicales en un 19.5\% (Ingle \& Taintor). A pesar de lo anteriormente señalado, y aun cuando la estructura normal de un canino, premolar mandibular y de un premolar maxilar se encuentra ampliamente definida, queda de manifiesto la importancia de realizar un correcto y completo examen clínico y de exámenes complementarios, ya que sólo de esta forma es posible establecer si un paciente se encuentra dentro o fuera de las cifras expuestas.

El clínico en el área de la odontología debe estar siempre atento a la posibilidad de encontrar variaciones internas y externas a la anatomía normal, y nunca asumir que el sistema de conductos de un diente es una entidad simple. Es importante considerar esta alteración de la morfología ante la presencia de piezas con patologías que requieran tratamientos de endodoncia o cualquier procedimiento al interior del sistema de conductos (D'Arcangelo, 2001; Holtzman), ya que la pertinente y adecuada indicación de exámenes complementarios, así como el análisis exhaustivo de ellos permite pesquisar oportunamente variaciones anatómicas de este tipo y puede evitar la ocurrencia de accidentes durante el desarrollo de procedimientos clínicos especialmente en las áreas de endodoncia y prótesis fija.

OPORTO, V. G. H.; FUENTES, F. R. E. \& SOTO, P. C. C. Tooth root and pulp canal anatomical variations. Int. J. Morphol., 28(3):945-950, 2010.

SUMMARY: Teeth development results from a complicated interaction between oral epithelium and underlying mesenchematic tissue during odontogenesis. Abnormal interaction between this tissues could result in development and eruption of an ectopical, anomalous teeth or with an anatomical variation. This report shows three cases of teeth with anatomical variations at root and pulp canal in mandibular canine and bicuspid, and maxillary first bicuspid. Diagnosis of these cases was performed through radiographical analysis. Mandibular canine and bicuspid shown two root and two pulp canals, maxillary first bicuspid presented three roots and three pulp canals. Considering that maxilar tooth showed caries closer to pulp chamber, patient was derived to an endodontic specialist to perform an endodontic treatment and rehabilitation. Idealizing root canal as a simple and invariable structure is a mistake. Clinicians always ought to take into consideration potential anatomical variations in teeth, especially in those that require endodontic treatments or any kind of procedure inside root canal system. Timely and adequate indication of imagenological complementary exams allow an adequate diagnosis of anatomical variations that will decrease chances of accidents during treatment.

KEY WORDS: anatomical variations, root canal, tooth roots.

\section{REFERENCIAS BIBLIOGRÁFICAS}

Abramovich, A. Histología y Embriología dentaria. $2^{\mathrm{a}}$ Ed. Buenos Aires, Panamericana, 1999.

D'Arcangelo, C.; Varvara, G. \& De Fazio, P. Root canal treatment in mandibular canines with two roots: a report of two cases. Int. Endod. J., 34(4):331-4, 2001.
Fuentes, F. R. \& Oporto, V. G. Tercer molar ectópico impactado en zona retromolar. Reporte de caso. Int. J. Morphol., 27:35-8, 2009.

Goh, Y. Ectopic eruption of maxillary molar tooth, an inusual cause of recurrent sinusitis. Singapore Med. J., 42:80-1, 2001. 
OPORTO, V. G. H.; FUENTES, F. R. E. \& SOTO, P. C. C. Variaciones anatómicas radiculares y sistemas de canales. Int. J. Morphol., 28(3):945-950, 2010.

Holtzman, L. Root canal treatment of a mandibular canine with three root canals. Case report. Int. Endod. J., 30:2913, 1997.

Ingle, J. \& Taintor, J. Endodoncia. México, Interamericana, 1987.

Ingle, J. \& Bakland, L. Endodoncia. $4^{\text {a }}$ Ed. México, McGraw-Hill Interamericana, 1996. pp.504-16.

Kitamura, H. Dental malformations and pathohistology. $1^{\text {st }}$ Ed. Ishiyaku, EuroAmerica, 1997.

Roig, M. \& Morelló, S. Introducción a la patología dentaria. Parte 1. Anomalías dentarias. Rev. Oper. Dent. Endod., 5:51, 2006.

Sharma, R.; Pccora, J.; Lumley, P. \& Kvalmsley, A. The external and internal anatomy of human mandibular canine teeth with two roots. Endod. Dent. Traumatol., 14:88-92, 1998.

Soares, I. \& Goldberg, F. Endodoncia. Técnicas y fundamentos. Buenos Aires, Panamericana, 2002. pp.26-9.

Soares, J. \& Leonardo, R. Root canal treatment of threerooted maxillary first and second premolars-a case report. Int. Endod J., 36:705-10, 2003.

Ten Cate, A. Histología Oral. $2^{\mathrm{a}}$ Ed. Buenos Aires, Panamericana, 1986.

Victorino, F.; Bernardes, R.; Baldi, J.; Moraes, I.; Bernardinelli, N.; Garcia, R. \& Bramante, C. Bilateral mandibular canines with two roots and two separate canals: case report. Braz. Dent. J., 20:84-6, 2009.
Dirección para correspondencia:

Gonzalo H. Oporto V.

Facultad de Medicina

Universidad de La Frontera

Manuel Montt 112

Temuco

CHILE

Email: goporto@ufro.cl

Recibido : 31-05-2010

Aceptado: 22-06-2010 